\title{
Sistema economico, impatto ambientale e benessere sociale: una lettura territoriale
}

\author{
SiLVIO Franco* Emanuele Blasi**
}

\begin{abstract}
Obiettivo del paper: Applicare un modello innovativo (stock e flussi) basato sul paradigma bioeconomico per identificare i sistemi economici locali, caratterizzarli in termini ambientali e sociali e cogliere le relazioni a scala territoriale fra sviluppo economico, benessere sociale e sostenibilità ambientale.

Metodologia: Individuazione del modello territoriale attraverso le componenti strutturali dei processi di produzione e consumo (stock) e i flussi in ingresso e uscita dal sistema. Quantificazione dei flussi con indicatori di benessere e sostenibilità ambientale; caratterizzazione degli stock con indicatori di dimensione e funzionalità produttiva.

Risultati: Il modello è stato applicato agli 8 sistemi economici locali della provincia di Viterbo. Partendo dalla valutazione degli indicatori di flusso e di stock sono state analizzate le relazioni fra sviluppo economico, benessere e sostenibilità ambientale a livello locale.

Limiti della ricerca: Studio basato su di un modello innovativo che richiede ulteriori validazioni. Soggettività nella scelta e nelle modalità di calcolo degli indicatori. Applicazione empirica su uno specifico caso di studio che limita le possibilità di generalizzazione dei risultati.

Implicazioni pratiche: Definizione di una metodologia strutturata finalizzata allo studio delle relazioni a livello locale fra sviluppo economico, benessere e sostenibilità ambientale. Disponibilità di dati per indirizzare specifiche azioni di sviluppo locale da parte delle istituzioni e del sistema delle imprese.

Originalità del lavoro: Approccio metodologico innovativo basato sul paradigma bioeconomico. Definizione di un modello integrato per lo studio e l'analisi quantitativa dei sistemi economici locali in una chiave territoriale. Evidenze empiriche scaturite dai risultati dell'applicazione del modello.
\end{abstract}

Parole chiave: sistemi economici locali; bioeconomia; modello stock e flussi; sviluppo locale; benessere eudaimonico; sostenibilità ambientale

Purpose of the paper: To apply an innovative model (stocks and flows) based on the bioeconomics paradigm with the aim to identify local economic systems, to characterize them

* Ricercatore di Economia e Gestione delle Imprese - Università della Tuscia e-mail.franco@unitus.it

** Dottore di Ricerca di Economia e Territorio - Università della Tuscia

e-mail: e.blasi@unitus.it 
in environmental and social terms and to gain insight into the relationships among economic development, social well-being and environmental sustainability at territorial level.

Methodology: Definition of the territorial model through the structural components of production and consumption processes (stocks) and system input and output (flows). Flows measurement by means of indicators of well-being and environmental sustainability; stocks measurement by means of indicators of productive dimension and functionality.

Findings: The model has been applied to the 8 local economic systems of Viterbo province. The interrelationships among economic development, well-being and sustainability at local level have been investigated starting from the evaluation of flows and stocks indicators

Research limits: The study is based on an innovative model that requires further validations. Indicators choice and evaluation is largely subjective. Difficulty in results generalization due to an empirical application referred to a specific case study.

Practical implications: Definition of a structured methodology suited to analyze the relationship among economic, social and environmental dimensions at local level. Data availability to address targeted actions towards local development carried out by local institutions and companies system.

Originality of the study: Innovative methodological approach based on bioeconomics paradigm. Definition of an integrated model to study and analyse, in quantitative terms, local economic systems in a territorial perspective. Empirical evidences derived from the application of stocks and flows model.

Key words: local economic system; bioeconomics; stocks and flows model; local development; eudaimonic well-being; environmental sustainability

\section{Introduzione: il paradigma bioeconomico e il modello "stock e flussi”}

La bioeconomia proposta da Nicholas Georgescu-Roegen costituisce un paradigma che include esplicitamente nella rappresentazione del sistema economico gli aspetti ambientali e sociali del contesto nel quale esso si sviluppa (GeorgescuRoegen, 2003).

Nel 1971 con la pubblicazione del saggio The entropy law and the economic process, la visione di Georgescu-Roegen si manifestò in modo organico; in questo scritto viene proposta una nuova teoria della produzione in cui la variabile tempo e le conseguenze del secondo principio della termodinamica sulla generazione di valore vengono introdotti come parametri fondamentali dei processi economici. Inserendo questi due concetti nella funzione di produzione, il principio della piena sostituibilità tra i capitali - economico e ambientale - coinvolti nel processo viene superato da una chiara distinzione tra agenti di trasformazione (fondi) e oggetti della trasformazione (flussi) tra di loro insostituibili. Il fondo riesce a "entrare e uscire dal processo con la propria efficienza immutata" (Georgescu Roegen, 1971, p.230) mentre il flusso, attraversando il confine del processo produttivo, viene utilizzato e trasformato in beni e prodotti e, pertanto, al termine del processo stesso non è più riconoscibile nelle sue caratteristiche originarie. 
Nonostante l'autore si sia concentrato nello studio del processo di produzione, nelle sue opere è evidente il tentativo di creare un'estensione dell'approccio bioeconomico anche per la sfera del consumo con l'obiettivo di uscire dal riduzionismo del binomio utilità-benessere proposto dalla teoria neoclassica (Zamagni, 1979).

Coerentemente con i presupposti epistemologici, quali la distinzione tra agenti (fondi) e oggetti (flussi) di trasformazione e la critica ai postulati di utilità, razionalità e non sazietà del consumatore, $\mathrm{i}$ concetti chiave della bioeconomia sono stati sviluppati in chiave sistemica nel modello a "stock e flussi" (S\&F). In tale modello viene introdotto il concetto di stock il quale, a differenza del fondo, possiede la capacità di modificare la sua struttura e la sua funzione al fine di preservare la propria esistenza (Bonaiuti, 2003). Questa rappresentazione degli stock fa riferimento alla teoria dei sistemi complessi, in particolare per quanto riguarda la capacità delle strutture, siano esse biologiche o sociali, di modificarsi per assicurare il proprio mantenimento attraverso un processo di continua riorganizzazione interna detto autopoiesi (Maturana e Varela, 1980).

Altro aspetto del modello $\mathrm{S} \& \mathrm{~F}$, direttamente ripreso dalla visione roegheriana, è quello di attribuire al sistema economico il fine di generare uno stato di eudaimonia per la collettività, intendendo con questo termine la capacità di creare le condizioni per il benessere duraturo dei membri della comunità di riferimento del sistema economico stesso. Lo stato di eudaimonia, oggetto delle riflessioni filosofiche di Aristotele ed Epicuro, identifica una società in cui ogni individuo percepisce la propria vita come degna di essere vissuta ossia dove le capacità di ciascuno trovano giusti tempi e spazi nei quali riuscire a svilupparsi, maturare ed esprimersi.

Nel modello $\mathrm{S} \& \mathrm{~F}$ il sistema economico è rappresentato da due sfere distinte, quella dei processi di produzione e quella dei processi di consumo, che, come schematizzato in figura 1, attraverso la loro azione congiunta sono responsabili della trasformazione delle risorse naturali in scarti e rifiuti e benessere duraturo per la popolazione (Bonaiuti, 2008; Blasi et al., 2008).

Entrambi i sistemi di produzione e consumo sono caratterizzati da degli stock (economici, ambientali, sociali, valoriali-istituzionali) i quali rappresentano gli agenti di trasformazione dei flussi in ingresso in flussi in uscita. Nello specifico, $\mathrm{i}$ processi di produzione operano una trasformazione in grado di attribuire alla materia/energia in uscita (prodotti) un valore più elevato della materia/energia in entrata (risorse naturali), con lo scopo di trasferire tale valore agli agenti di trasformazione (capitale e lavoro). È opportuno precisare che i flussi in uscita dal sistema di produzione sono costituiti, oltre che dai prodotti che rappresentano i flussi in ingresso dei processi di consumo, anche dagli scarti e dai rifiuti generati dai processi, in particolare di tipo agricolo e industriale. In modo analogo, il processo di consumo, attraverso i suoi stock, trasforma materia ed energia ad alta utilità, inglobata nei beni in uscita dalla sfera della produzione, in materia ed energia a bassa utilità (scarti e rifiuti) allo scopo di soddisfare i bisogni della collettività. 
Fig. 1: Il processo economico nel modello stock e flussi

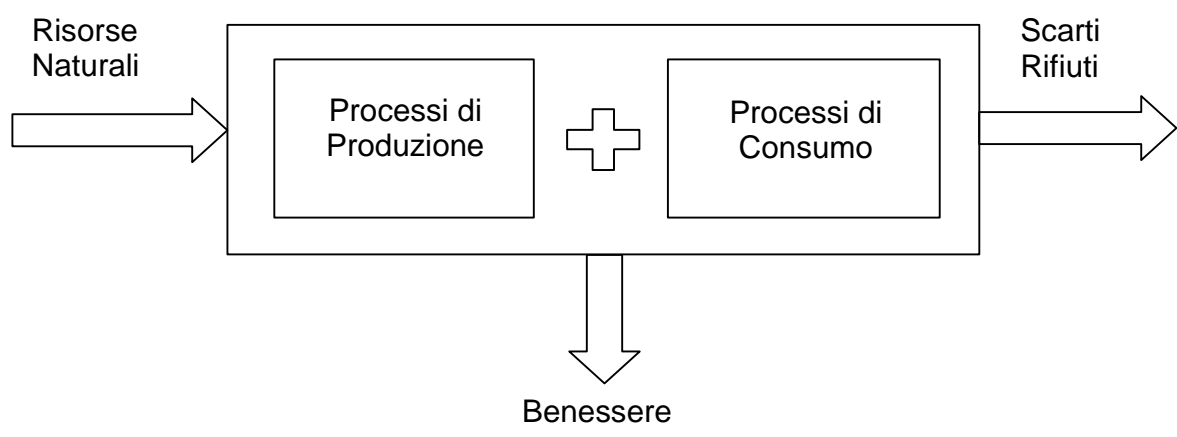

Fonte: ns. elaborazione

L'approccio al sistema economico condotto attraverso questo paradigma consente di studiare, secondo un approccio teoricamente coerente, le relazioni che legano la dimensione strutturale del sistema economico con la sua finalità di generare benessere nella collettività e con la capacità degli ecosistemi di sostenerne il funzionamento in termini di disponibilità di risorse naturali e di assorbimento degli scarti. Lo svolgimento di un tale studio, soprattutto qualora se ne voglia tentare una declinazione in termini empirici, impone che il modello S\&F venga riferito a una esplicita scala territoriale capace di esprimere una dimensione economica, intesa come insieme di processi di produzione e consumo, una dimensione sociale, identificabile in una ben definita comunità di riferimento, e una dimensione ambientale, caratterizzabile in termini dei servizi che gli ecosistemi locali sono in grado di assicurare.

Partendo da questo approccio teorico, il presente lavoro si pone l'obiettivo di caratterizzare le relazioni tra sistema produttivo, impatto ambientale e benessere della collettività a livello territoriale. A questo scopo si affronterà la caratterizzazione dei flussi in entrata e uscita dal sistema, attraverso la definizione di indicatori atti a valutare la consistenza del benessere e della sostenibilità ambientale in un territorio, e la descrizione della dimensione e della funzionalità degli stock coinvolti nei processi di produzione, con particolare riferimento al capitale propriamente inteso e alla struttura sociale sottesa all'organizzazione ed all'utilizzo del lavoro. Successivamente si procederà ad individuare la scala con cui, applicando il modello S\&F, è possibile analizzare le relazioni fra la dimensione del sistema economico, il benessere e l'impatto ambientale. La definizione degli indicatori e della scala territoriale sarà utilizzata per condurre un'applicazione empirica che, al di là dei risultati ottenuti, consentirà di valutare le concrete potenzialità di applicazione del modello allo studio in chiave territoriale delle relazioni fra economia, ambiente e società. 


\section{Indicatori di flusso del sistema locale}

\subsection{II flusso di benessere}

Il riferimento al modello S\&F, che vede nella generazione di eudaimonia il fine ultimo del sistema economico, richiede una definizione di questa forma di benessere che possa essere tradotta in un indicatore in grado di esprimerne il livello quantitativo. Considerando che il benessere eudaimonico identifica una condizione nella quale il contesto (economico, sociale, ambientale) crea le condizioni affinché gli individui percepiscano la loro vita come degna di essere vissuta, il percorso di misurazione di questo flusso pone almeno due difficoltà di ordine pratico. La prima è legata alla sua connotazione di subjective well-being, che ne impedisce la valutazione sulla base di dimensioni oggettive, come invece accade nelle indagini finalizzate alla misurazione della qualità della vita o del welfare che utilizzano dati statistici secondari. Allo stesso tempo, e questa rappresenta la seconda difficoltà, non è possibile utilizzare strumenti d'indagine propri della ricerca medica e psicologica, in quanto questi mirano a determinare il benessere edonico, vale a dire lo stato temporaneo di felicità/infelicità individuale, che è influenzato in larga misura dalle caratteristiche individuali e dalle vicende personali e solo marginalmente dalle condizioni del contesto socioeconomico ed ambientale.

La prospettiva eudaimonica, al contrario, individua come fattore fondamentale la relazione tra benessere del singolo e sviluppo della collettività; in questa chiave vengono enfatizzate le capacità di perseguire obiettivi significativi per il singolo e la società, la mobilizzazione delle risorse, l'incremento delle abilità e dell'autonomia individuale, le competenze sociali e il ruolo delle relazioni nella promozione dell'individuo e della comunità (Blasi et al., 2012). In questa prospettiva Ryan e Deci (2000), attingendo alla teoria dei bisogni di Maslow (1943), sostengono che l'uomo nasce con bisogni psicologici specifici che spiegano la sua motivazione a sviluppare propri interessi e capacità". Sarà quindi interesse dei singoli, proprio per creare le condizioni che consentono di dare un significato alla propria vita, contribuire a realizzare una società in cui sono tenuti in alta considerazione $\mathrm{i}$ valori sociali, ambientali, relazionali e culturali. Questa condizione permette di guardare al benessere come una condizione individuale che è, allo stesso tempo, la base del miglioramento del benessere diffuso nella società.

In questo studio, per esprimere una valutazione del livello di eudaimonia di un gruppo di individui, si propone l'utilizzo dell'indagine diretta che, attraverso un questionario, valuti la loro percezione rispetto alle dimensioni riconducibili a questa accezione di benessere e identificabili in: auto-accettazione; capacità di seguire e realizzare le convinzioni personali; relazioni positive con gli altri; appagamento bisogni materiali; posizionamento sociale (Ryff, 1989; Diener e Suh, 1997).

Per arrivare alla definizione di una misura rispetto a queste 5 dimensioni sono stati formulati 15 item basandosi su quanto proposto in diversi studi finalizzati a cogliere le percezioni individuali del subjective well-being e a valutarne, con una prospettiva più o meno esplicita, la caratterizzazione eudaimonica (Ryff, 1989; 
Endicott et al., 1993, Ruini et al., 2003, Blasi et al., 2012). Ciascun item prevede una risposta strutturata con una scala Likert a 5 livelli che esprime il grado di accordo dell'intervistato con la relativa affermazione (l'elenco completo degli item presenti nel questionario è riportato in Appendice). Il livello di benessere percepito può essere ottenuto sia mediando le risposte ai 15 item, sia valutando la media per ciascuna dimensione e poi mediando i cinque valori ottenuti. Con questa seconda modalità, è possibile assegnare a ciascuna dimensione un peso diverso in funzione dell'importanza ad essa implicitamente attribuita dall'insieme degli intervistati. Tale sistema di ponderazione prevede che il peso di ogni dimensione sia assegnato tenendo conto del legame medio che si riscontra ex-post fra i punteggi delle singole dimensioni e il livello di accordo con l'affermazione (di controllo) inserita come item numero 16 nel questionario "nell'insieme sono soddisfatto della qualità della mia vita". A questo scopo, partendo da un sufficiente numero di rilevazioni, è possibile impostare un modello di regressione lineare multipla in cui il livello complessivo di soddisfazione per la propria vita percepito dal singolo intervistato viene spiegato come combinazione lineare del punteggio che egli ha attribuito alle 5 dimensioni. I coefficienti ottenuti dall'applicazione del modello all'insieme dei dati rilevati con i questionari possono essere utilizzati per determinare il livello del benessere individuale come combinazione del valore soggettivamente alle diverse dimensioni ponderate secondo il sistema di pesi espresso dall'insieme del gruppo cui appartiene l'individuo stesso.

Per determinare il livello di eudaimonia di una comunità è necessario trovare un'adeguata modalità di aggregazione dei valori individuali espressi dal campione analizzato, il quale deve essere selezionato in modo da risultare rappresentativo della comunità stessa. Il parametro più adatto a questo scopo è la mediana, la misura di tendenza centrale che risulta meno influenzata dai valori estremi, che in questo caso potrebbero essere originati da situazioni individuali legate a momentanee circostanze di felicità o dolore.

\subsection{I flussi di risorse naturali e la capacità di carico degli ecosistemi}

Seguendo la lettura rohegeriana, l'effettiva capacità di un sistema economico di creare uno stato di eudaimonia è vincolata alla sua sostenibilità di lungo periodo. Tale condizione è assicurata dalla continuità degli stock di operare quali agenti di trasformazione e dell'ambiente di fornire i flussi di materia ed energia in ingresso e assorbire i flussi di scarti e rifiuti in uscita. Laddove questi presupposti non fossero rispettati, il livello di benessere osservato nella comunità, anche laddove risultasse molto elevato, non esprimerebbe uno stato di eudaimonia in quanto verrebbero a mancare le condizioni che ne garantiscono il mantenimento.

Con riferimento alla dimensione ambientale, l'adozione del modello S\&F richiede di confrontare, su base territoriale, l'entità dell'insieme dei flussi in input, sotto forma di materia ed energia necessaria per l'attivazione e il mantenimento dei processi di produzione e consumo, e di output, sotto forma di scarti dei processi di 
produzione e di consumo, con la disponibilità di risorse naturali e la capacità di assorbimento dei rifiuti.

Per operare questo confronto è stato utilizzato il metodo del Bilancio Ecologico che permette di operare una stima dell'impatto ambientale delle attività che insistono su un territorio rispetto alla sua capacità di carico (carrying capacity). Tale bilancio è basato sul confronto di due indicatori noti come Impronta Ecologica e Biocapacità. Il primo quantifica la domanda di risorse espressa dalle attività che hanno luogo sul territorio, il secondo esprime la disponibilità complessiva di risorse presenti nel territorio stesso. La differenza fra Biocapacità e Impronta Ecologica definisce il Bilancio Ecologico: a un valore negativo (positivo) corrisponde una situazione di deficit (surplus) ecologico, ovvero una condizione di insostenibilità (sostenibilità) in cui i consumi di risorse naturali risultano superiori (inferiori) ai livelli di rigenerazione da parte degli ecosistemi locali. L'entità del surplus (deficit) ecologico rappresenta una stima del livello di sostenibilità (insostenibilità) dello stile di vita di una collettività rispetto alle risorse dell'area presa come riferimento spaziale, nel nostro caso il territorio del sistema locale.

L'Impronta Ecologica, introdotta da Rees e Wackernagel (1994; 2008), quantifica l'area di ecosistemi (terrestri e acquatici) necessari per la fornitura delle risorse utilizzate e l'assorbimento delle emissioni prodotte; tale indicatore, misurato in ettari globali (global hectares - gha), esprime quindi la richiesta di capitale naturale da parte di una popolazione. Il concetto di Impronta Ecologica si basa sull'idea che a ogni unità di materia o di energia che entra nel sistema economico corrisponde una certa estensione di territorio che garantisce il relativo apporto di risorse e il conseguente assorbimento di rifiuti. L'indicatore Biocapacità misura l'offerta di produttività biologica fornita da terre coltivate, pascoli, foreste, aree marine produttive e, in parte, aree edificate o in degrado, anch'essa espresso in gha.

L'elasticità della metodologia permette una piena adattabilità a diverse dimensioni spaziali e può quindi essere utilizzata per condurre analisi con riferimento a differenti unità territoriali. Dalle prime analisi a livello nazionale (Wackernagel e Rees, 1996) si è passati, anche per quanto riguarda l'Italia, a studi a scala regionale (Wwf Italia et al., 2002; Ministero dell'Ambiente e WWF, 2004), provinciale (Provincia di Milano, 2008; Bagliani et al., 2008; Blasi e Passeri, 2009) fino alla dimensione di nucleo urbano (Scotti et al., 2009).

\section{Scala territoriale e dimensione produttiva del sistema economico}

\subsection{La scala territoriale per la definizione del sistema economico locale}

Definiti gli indicatori per la misurazione dei flussi del sistema economico, in questo paragrafo si propone una scala territoriale per l'applicazione del modello $\mathrm{S} \& \mathrm{~F}$ che sia coerente con la teoria di riferimento e che consenta lo svolgimento di applicazioni empiriche che mirano a studiare le relazioni fra la struttura produttiva del sistema economico, la sua sostenibilità ambientale e la capacità di generare 
benessere nella comunità locale. Ne deriva che la scala territoriale per l'analisi del sistema economico locale deve comprendere al suo interno un numero rilevante di differenti tipologie di processi di produzione e consumo, accogliere una comunità che condivide il sistema di valori alla base della percezione del benessere e includere degli ecosistemi identificabili nella loro capacità biologica di fornire risorse e assorbire rifiuti.

Delle aree che sembrano possedere in misura soddisfacente tali caratteristiche sono i Sistemi Locali del Lavoro (SLL), determinati come risultato di una zonizzazione funzionale sviluppata dall'ISTAT sulla base delle rilevazioni censuarie sui flussi giornalieri di pendolarismo (ISTAT e IRPET, 1989). I SLL sono delle aggregazioni di comuni contigui i cui confini sono definiti in modo da contenere una quota prefissata (che nell'ultimo censimento è stata posta pari al 75\%) degli spostamenti tra luogo di residenza e luogo di lavoro degli occupati locali. Si parla di SLL, quindi, come area di "autocontenimento del lavoro", intendendo con questo termine che in quel territorio la gran parte dei lavoratori residenti trova la propria occupazione e, allo stesso tempo, $\mathrm{i}$ posti di lavoro disponibili sono coperti in misura largamente predominante dalla popolazione locale. La modalità di definizione dei SLL e la coerenza dell'algoritmo utilizzato per la loro delimitazione consente di pervenire alla definizione di un reticolo territoriale individuato da unità territoriali "statisticamente e geograficamente significative per l'analisi di fenomeni economici e sociali" (OECD, 2002).

Proprio in conseguenza della loro modalità di definizione, i SLL identificano dei territori all'interno dei quali i residenti svolgono le loro attività e, di conseguenza, trascorrono la maggior parte del proprio tempo. Ne consegue che nei SLL si concentrano la maggiore quantità possibile delle relazioni individuali che intervengono tra luoghi dove si svolgono attività di produzione di beni e servizi e luoghi dove si esercitano le attività legate al contesto individuale, familiare e sociale (Barbieri e Pellegrini, 2001). I SLL sembrano quindi adattarsi in modo soddisfacente alla lettura territoriale operata dal modello $\mathrm{S} \& \mathrm{~F}$, sia per quanto riguarda la presenza e l'identificazione degli stock che operano come agenti di trasformazione nei processi di produzione e di consumo, sia come sede di una comunità costituita da individui che condividono le dinamiche quotidiane legate alla residenza, al lavoro e alle relazioni sociali. Inoltre, la dimensione sovracomunale e il vincolo di contiguità spaziale dei SLL consentono di circoscrivere porzioni di territorio in grado di contenere degli ecosistemi naturali capaci di fornire servizi ambientali per lo svolgimento delle attività che vi hanno luogo. Tale prerequisito permette di verificare in che misura il territorio è capace di offrire risorse capaci di soddisfare nel lungo periodo le richieste del sistema economico.

In questo lavoro, per riuscire a verificare l'esistenza di tendenze che legano le performance dei processi di produzione e di consumo con la capacità di creazione di condizioni di benessere duraturo, si utilizzerà una metodologia che prevede il confronto di diversi SLL in termini di dimensione degli stock produttivi, flusso di benessere e sostenibilità ambientale. Tale approccio si basa sull'ipotesi che i sistemi stessi possiedano una struttura abbastanza simile per quanto riguarda gli altri 
elementi non esplicitamente considerati nell'analisi, in particolare gli stock sociali e valoriali relativi alla sfera del consumo; la loro eterogeneità porterebbe, infatti, a una diversa percezione nell'immaginario del benessere rendendo poco agevole la fase di comparazione fra i sistemi locali e limitando la possibilità di interpretazione dei risultati.

\subsection{La dimensione strutturale del sistema produttivo locale}

Dopo aver definito gli indicatori di flusso e la dimensione territoriale del sistema locale, rimane da affrontare la questione di come caratterizzarne in termini quantitativi la dimensione dello stock di produzione. $\grave{E}$ questo un compito certamente non facile, che pone problemi di tipo teorico e metodologico la cui trattazione va ben oltre i fini di questo studio. Ciò che si può affermare è che la valutazione della dimensione strutturale del sistema di produzione richiede, secondo la logica del modello S\&F, l'utilizzo di indicatori di tipo fisico, capaci di definire sia l'effettiva capacità di trasformazione di energia e materia (flusso in ingresso) in prodotti e scarti (flusso in uscita), sia la consistenza legata al capitale economico e sociale preposto a tale funzione. Si tratta, in altri termini, di quantificare la solidità del sistema produttivo, la quale, nel nostro modello, è rappresentata dalla capacità degli stock di produzione di mantenere e sviluppare la propria consistenza, in particolare per quanto riguarda il capitale (materiale e immateriale) e le strutture sociali del lavoro. Per valutare questa dimensione ci si può riferire alla generazione di valore da parte del sistema delle imprese come espressione della funzionalità strutturale e operativa - degli stock impiegati nei processi di produzione.

Un indicatore in grado di quantificare tale dimensione, seppure in modo approssimato e parziale, è il valore aggiunto pro capite; questo, infatti, rappresenta una proxy di quanto il sistema produttivo sia in grado di generare e trasferire valore agli stock presenti sul territorio. Tale affermazione è giustificata dal fatto che la proprietà dell'autocontenimento del mercato del lavoro che caratterizza i SLL, fa si che gran parte degli occupati nelle imprese locali, datori di lavoro e dipendenti, siano residenti all'interno dello stesso territorio. Ne consegue che una quota rilevante del valore aggiunto generato dalle imprese del sistema locale viene distribuito fra soggetti che lavorano e vivono al suo interno e, di conseguenza, il suo ammontare per residente fornisce un'indicazione della capacità del sistema produttivo di sviluppare strutture, risorse umane e contesto sociale di riferimento.

\section{Economia, benessere e ambiente nei sistemi locali della provincia di Viterbo}

\subsection{I sistemi economici locali della provincia di Viterbo}

Allo scopo di sviluppare una caratterizzazione empirica dei sistemi locali in termini economici, ambientali e sociali e di descrivere il ruolo che il sistema di 
produzione può svolgere per contribuire al loro sviluppo, è stata condotta un'applicazione del modello S\&F con riferimento alla provincia di Viterbo.

Come precisato nel paragrafo precedente, alla luce del quadro teorico di riferimento e delle finalità dell'analisi, è possibile considerare come sistemi economici locali i SLL che insistono su tale territorio. La validità di tale scelta, nel caso della provincia di Viterbo, è corroborata da alcuni aspetti che rendono più "robusta" questa forma di zonizzazione; infatti, i SLL viterbesi, oltre a essere tutti contenuti nel territorio provinciale, hanno mantenuto pressoché stabile la loro configurazione nel corso degli ultimi 30 anni, mostrando una notevole capacità descrittiva della struttura territoriale della provincia dal punto di vista socioeconomico.

Le ragioni di tale stabilità sono da ricercare nell'inerzia sociale e nello scarso spirito imprenditoriale che, per ragioni storiche, caratterizzano la provincia di Viterbo e che hanno causato la sua marginalizzazione dai processi di sviluppo industriale e infrastrutturale che hanno riguardato le regioni limitrofe (bassa Toscana, Umbria e Marche) portando alla nascita della cosiddetta "terza Italia" (Franco e Senni, 2002). Questa situazione, se da un lato ha favorito la conservazione dei paesaggi storici e ambientali anche a ridosso dei centri abitati, dall'altro ha condizionato buona parte delle attività economiche mantenendole, se si esclude il distretto industriale di Civita Castellana, a una dimensione limitata alle richieste del mercato locale. Ciò ha determinato il mantenimento di un tessuto agricolo che conferisce alla provincia di Viterbo una connotazione rurale molto più accentuata delle altre province laziali e di quelle confinanti e che la pone ai primi posti in Italia in termini di grado di ruralità (Angeli et al., 2001).

L'insieme di questi fattori ha contribuito a mantenere una dimensione sociale e valoriale abbastanza uniforme all'interno del territorio provinciale e, allo stesso tempo, una sostanziale stabilità delle dinamiche residenziali e occupazionali; proprio questo secondo aspetto è alla base del fatto che, come già detto, i SLL viterbesi hanno mantenuto in larga misura invariati nel tempo i loro confini e quindi individuino delle comunità ben definite e stabili nel tempo.

Nella figura 2 è riportata la configurazione territoriale dei SLL della provincia di Viterbo così come elaborata dall'Istat tenendo conto dei relativi dati costitutivi determinati sulla base dei risultati del censimento della popolazione del 2001 (Istat, 2005). La tabella 1 riporta il valore di tali dati costitutivi preceduto, per ciascun SLL, dalla popolazione residente al 31-12-2011 (Istat, 2012).

Senza entrare nel merito dei dati riportati in tabella, è opportuno evidenziare due elementi. Il primo riguarda la diffusa incapacità dei sistemi locali viterbesi di offrire un sufficiente numero di posti di lavoro agli occupati residenti. Nello specifico, con riferimento ai dati del 2001, si osserva come oltre 7.000 occupati residenti nella provincia svolgano la propria attività lavorativa al di fuori di essa. Il secondo aspetto, più interessante per i nostri scopi, riguarda gli elevati livelli di autocontenimento della domanda e, in misura leggermente più ridotta, dell'offerta, i quali confermano la "solidità" strutturale dei sistemi locali e, quindi, la loro configurazione sufficientemente definita di comunità economica e sociale. 
Fig. 2: I SLL della provincia di Viterbo

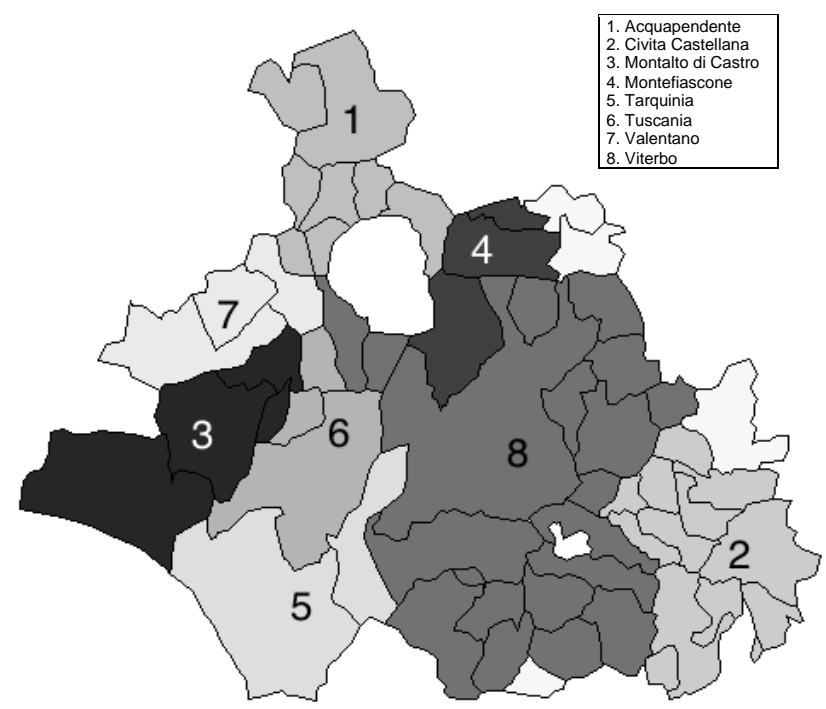

Fonte: ns. rappresentazione da dati Istat, 2005

Tab. 1: Residenti, dati costitutivi e autocontenimento del lavoro nei SLL di Viterbo

\begin{tabular}{|lcccccc|}
\hline Nome SLL & $\begin{array}{c}\text { Residenti } \\
(2011)^{a}\end{array}$ & $\begin{array}{c}\text { Posti di } \\
\text { lavoro }^{b}\end{array}$ & $\begin{array}{c}\text { Occupati } \\
\text { resident }^{b}\end{array}$ & $\begin{array}{c}\text { Spostamenti } \\
\text { interni }^{b}\end{array}$ & $\begin{array}{c}\text { Autocont. } \\
\text { Domanda }^{b}\end{array}$ & $\begin{array}{c}\text { Autocont. } \\
\text { Offerta }^{b}\end{array}$ \\
\hline Acquapendente & 18.987 & 4.006 & 4.662 & 3.574 & $89,2 \%$ & $76,7 \%$ \\
Civita Castellana & 65.574 & 13.177 & 15.713 & 11.057 & $83,9 \%$ & $70,4 \%$ \\
Montalto di Castro & 15.933 & 3.467 & 3.672 & 2.838 & $81,9 \%$ & $77,3 \%$ \\
Montefiascone & 18.331 & 3.696 & 4.575 & 2.898 & $78,4 \%$ & $63,3 \%$ \\
Tarquinia & 18.718 & 4.149 & 4.816 & 3.464 & $83,5 \%$ & $71,9 \%$ \\
Tuscania & 11.392 & 1.986 & 2.692 & 1.677 & $84,4 \%$ & $62,3 \%$ \\
Valentano & 7.054 & 1.210 & 1.710 & 1.017 & $84,1 \%$ & $59,5 \%$ \\
Viterbo & 147.360 & 34.495 & 35.365 & 29.028 & $84,2 \%$ & $82,1 \%$ \\
\hline
\end{tabular}

Fonte: ${ }^{a}$ Istat, $2012 ;^{b}$ Istat, 2005

\subsection{La dimensione economica, sociale e ambientale nei sistemi locali}

Con riferimento agli 8 sistemi locali della provincia di Viterbo sono stati valutati gli indicatori relativi agli stock produttivi (dimensione economica), al bilancio ecologico fra flussi di risorse naturali e di scarti e rifiuti, da un lato, e servizi ecosistemici dall'altro (dimensione ambientale) e al flusso di benessere eudaimonico (dimensione sociale).

La dimensione economica del sistema locale, seppure con i limiti cui si è fatto cenno nel paragrafo precedente, viene descritta attraverso l'entità del valore 
aggiunto pro capite. Questo indicatore è disponibile, già a livello di SLL, in uno studio dell'Istat che ne ha elaborata la serie storica nel periodo 2001-2005 (Istat, 2008); successivamente non sono state condotte ulteriori valutazioni di tale indicatore, pertanto il 2005 è l'anno di riferimento dei valori riportati nella prima colonna della tabella 2.

La quantificazione del flusso di benessere generato dal sistema è espressa da un indicatore sintetico la cui valutazione è stata eseguita attraverso lo svolgimento di un'indagine diretta. Questa è stata basata su un campionamento a strati definito sulla struttura socio-demografica dei singoli SLL in termini di sesso, età e comune di residenza. Per ottenere una copertura sufficientemente omogenea delle diverse componenti socio-demografiche della popolazione, si è posta attenzione ad identificare i luoghi e gli orari in cui effettuare le interviste, tenendo conto delle caratteristiche dei diversi SLL. L'indagine è stata svolta da Marzo a Giugno 2010 e ha coinvolto complessivamente 10 intervistatori a cui sono state attribuite delle specifiche aree di rilevazione. Il questionario utilizzato, come già descritto, ha previsto la risposta a 16 item, 15 dei quali coprono le 5 dimensioni individuate per la valutazione del benessere eudaimonico cui si aggiunge un item di controllo, oltre alla specifica di alcuni dati demografici dell'intervistato. Nel complesso sono state svolte oltre 1.150 interviste (pari a circa lo $0,5 \%$ della popolazione provinciale compresa fra i 20 e gli 80 anni); la cura nella proposizione del questionario, le indicazioni chiaramente riportate nel testo e la compilazione eseguita con la possibilità di rivolgersi all'intervistatore, hanno ridotto drasticamente il numero di questionari incompleti o inutilizzabili. Per la rilevazione del livello di benessere su base territoriale sono stati considerati esclusivamente i questionari compilati da intervistati che rispondevano alla condizione di appartenenza ai SLL in termini di residenza e occupazione. Si è così arrivati a un campione di 972 osservazioni il quale ha rappresentato il riferimento per la valutazione del flusso di benessere prodotto dai sistemi economici locali della provincia di Viterbo, il cui valore è riportato nella seconda colonna della tabella 2 (Blasi et al., 2012).

Infine, per quanto riguarda la sfera ambientale, l'indicatore di riferimento è basato sul confronto fra la domanda di servizi ambientali del sistema economico locale e l'offerta di tali servizi da parte del territorio su cui insiste il sistema stesso. Come si è visto, la domanda di servizi ambientali, espressa dalla richiesta di materia/energia utilizzata dai processi di produzione - flusso in ingresso - e dalla capacità di assorbire gli scarti e i rifiuti prodotti da imprese e consumatori - flusso in uscita -, può essere quantificata attraverso l'Impronta Ecologica. Considerando la difficoltà di operare un calcolo affidabile di questo indicatore a livello subprovinciale si è considerato un medesimo valore di Impronta Ecologica per tutti i sistemi locali. Per quanto riguarda l'offerta dei servizi ambientali, invece, è stata valutata la Biocapacità a livello comunale, ottenendo un diverso livello per ciascuno dei SLL (Blasi e Passeri, 2009). Così facendo si è ipotizzato che i flussi di input (materia ed energia) e di output (scarti e rifiuti) pro capite siano gli stessi in ciascun SLL, mentre i servizi ecosistemici si differenzino in relazione alle caratteristiche ambientali e alla gestione del territorio operata a livello locale (Blasi et al., 2010). 
La differenza fra Biocapacità e Impronta Ecologica stabilisce il Bilancio Ecologico, indicatore di sostenibilità del sistema economico locale, la cui entità è riportata nell'ultima colonna della tabella 2.

Tab. 2: Indicatori di performance economica, sociale e ambientale dei sistemi locali viterbesi

\begin{tabular}{|lccc|}
\hline Sistema Economico Locale & $\begin{array}{c}\text { Valore Aggiunto pro } \\
\text { capite }(€)^{a}\end{array}$ & $\begin{array}{c}\text { Livello di benessere } \\
(1-5)^{b}\end{array}$ & $\begin{array}{c}\text { Bilancio ecologico } \\
(\text { gha })^{c}\end{array}$ \\
\hline 1. Acquapendente & 12.450 & 3,28 & 2,25 \\
2. Civita Castellana & 16.600 & 3,35 & $-0,95$ \\
3. Montalto di Castro & 19.230 & 3,31 & 2,64 \\
4. Montefiascone & 13.670 & 3,22 & $-0,47$ \\
5. Tarquinia & 15.020 & 3,47 & 0,81 \\
6. Tuscania & 10.810 & 3,13 & 3,12 \\
7. Valentano & 10.910 & 3,19 & 4,24 \\
8. Viterbo & 22.510 & 3,27 & $-0,67$ \\
\hline
\end{tabular}

Fonte: ${ }^{a}$ Istat, 2008; ${ }^{\mathrm{b}}$ Blasi et al., 2012; ${ }^{\mathrm{c}}$ Blasi e Passeri, 2009

\subsection{Le relazioni osservate fra le dimensioni}

Pur nella consapevolezza che gli indicatori scelti presentano dei limiti, sia nella loro confrontabilità temporale, sia nella capacità di descrizione delle condizioni economiche, sociali e ambientali dei sistemi locali, è interessante indagare il verso e l'intensità della loro associazione per evidenziarne e comprenderne le eventuali relazioni.

Un primo passo in questa direzione può essere compiuto attraverso un'analisi di correlazione, i cui risultati sono mostrati in tabella 3.

Tab. 3: Coefficienti di correlazione fra i tre indicatori

\begin{tabular}{|lccc|}
\hline & $\begin{array}{c}\text { Economica } \\
\text { (Valore aggiunto) }\end{array}$ & $\begin{array}{c}\text { Sociale } \\
\text { (Benessere) }\end{array}$ & $\begin{array}{c}\text { Ambientale } \\
\text { (Bil. ecologico) }\end{array}$ \\
\hline $\begin{array}{l}\text { Economica } \\
\text { (Valore aggiunto) }\end{array}$ & 1 & & \\
$\begin{array}{l}\text { Sociale } \\
\text { (Benessere) }\end{array}$ & 0,414 & 1 & 1 \\
$\begin{array}{l}\text { Ambientale } \\
\text { (Bil. Ecologico) }\end{array}$ & $-0,574$ & $-0,433$ & \\
\hline
\end{tabular}

Fonte: ns. elaborazioni

Le indicazioni che emergono da tale analisi sono abbastanza prevedibili, in particolare per quanto riguarda il legame della sostenibilità ambientale e del livello benessere con la performance economica dei sistemi locali.

Nel primo caso si osserva un'associazione negativa, la quale conferma come, all'interno di un territorio, l'incremento dimensionale della struttura produttiva si 
accompagni a un più intenso utilizzo delle risorse naturali e quindi a una minore sostenibilità ambientale.

Allo stesso modo, non desta sorpresa il legame positivo fra dimensione economica e sociale, la quale evidenzia come, in termini generali, all'aumentare della ricchezza distribuita si osservi un incremento del benessere sociale. Tale legame, tuttavia, oltre a non essere particolarmente intenso - l'aumento della ricchezza distribuita spiega solo il $17 \%$ del miglioramento della condizione di benessere - assume un andamento tutt'altro che lineare. Infatti, come si osserva dal grafico di figura 3, nei sistemi locali esaminati la relazione fra ricchezza e benessere è caratterizzata da un duplice andamento. Per bassi valori del valore aggiunto pro capite l'incremento della performance economica si associa a un significativo aumento del benessere; viceversa, oltre una certa soglia, l'aumento di ricchezza si traduce in una progressiva riduzione del livello del flusso di eudaimonia. Tale andamento è descritto dalla curva a $U$ rovesciata riportata nel grafico, ottenuta come prodotto di una regressione non lineare basata su una funzione polinomiale di secondo grado $\left(r^{2}=0,632\right)$. Questo risultato, con tutti i limiti del caso, rappresenta una conferma del paradosso di Easterlin, il quale, dimostrando come oltre un certo livello l'ulteriore crescita della ricchezza determini una riduzione del benessere (Easterlin, 1974), ha posto le basi teoriche per le critiche, ormai ampiamente condivise, al PIL quale unico indicatore della qualità della vita individuale e sociale.

Fig. 3: Relazione fra valore aggiunto pro capite e benessere nei sistemi locali

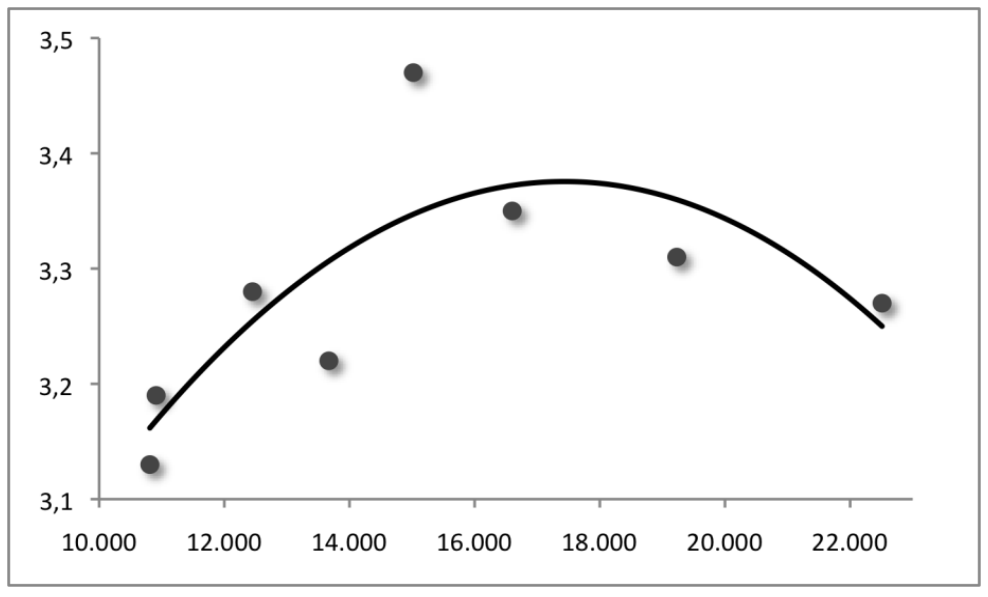

Fonte: ns. elaborazioni

Interessante è anche lo studio della relazione fra dimensione ambientale e sociale che, pur evidenziando un'associazione tendenzialmente negativa, mostra un andamento non uniforme. Infatti, come si osserva dalla figura 4, questo legame, che appare evidente per quei sistemi che si collocano in condizioni di un'ampia 
dotazione di risorse naturali non utilizzate a fini produttivi, sembra invertirsi quando i territori sono caratterizzati da situazioni di insostenibilità. La parte crescente della curva ad $U$ rovesciata sta proprio a indicare come una domanda di risorse naturali da parte del sistema produttivo che vada oltre la capacità di carico del territorio si traduca in una minore condizione di benessere della comunità locale. Fra l'altro, il fatto che la curva mostri il suo massimo nella regione che corrisponde a una condizione in cui l'utilizzo di risorse è leggermente inferiore alla loro disponibilità può suggerire, in termini del tutto indicativi, che la percezione del benessere individuale non prescinde dalla consapevolezza - più o meno esplicita - di un equilibrio efficiente fra funzionamento del sistema economico e qualità dell'ambiente.

Fig. 4: Relazione fra sostenibilità ambientale e benessere nei sistemi locali

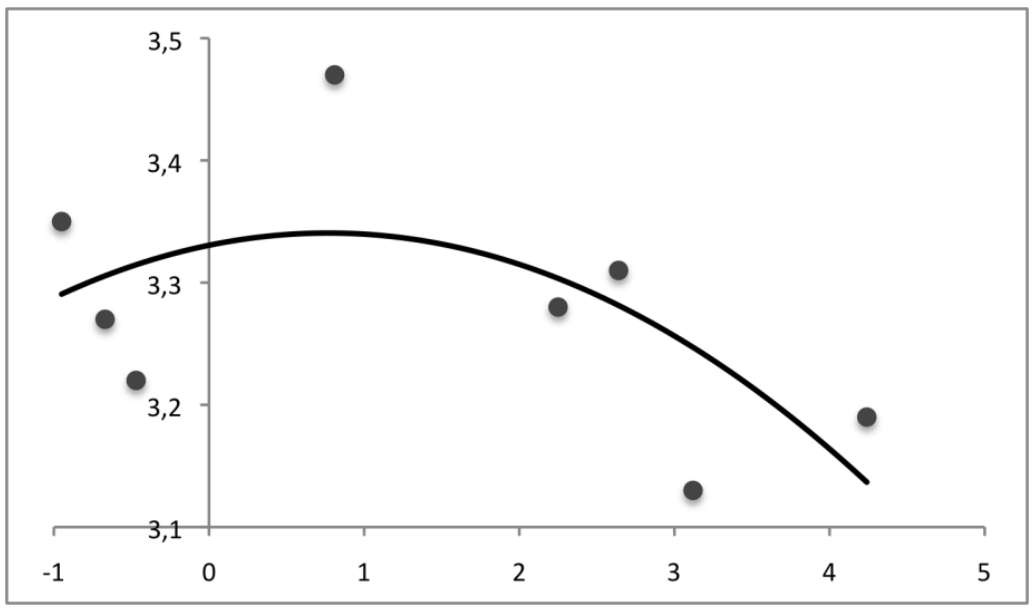

Fonte: ns. elaborazioni

\section{Considerazioni conclusive}

In questo lavoro si è tentato di affrontare il tema delle complesse relazioni che esistono fra sfera economica, sociale e ambientale attraverso una loro lettura territoriale a scala locale.

Il primo passo è consistito nell'individuazione di un paradigma teorico di riferimento che consentisse di analizzare congiuntamente le tre dimensioni, integrandole all'interno del medesimo riferimento spaziale. Questo è stato identificato con l'approccio bioeconomico che, secondo quanto proposto da Georgescu-Roegen, vede il sistema economico nel suo insieme come un agente di trasformazione che attraverso dei processi fisici degrada risorse naturali (materia ed energia) per creare benessere per gli esseri umani. 
Partendo da questo approccio epistemologico è stato definito un modello capace di calare questa visione del sistema economico in una dimensione maggiormente operativa. Tale modello, detto a "stock e flussi", che costituisce una variante di quello a "fondi e flussi" proposto dallo stesso Georgescu-Roegen, consente di operare una caratterizzazione degli agenti di trasformazione (stock) del sistema economico e degli input e output (flussi) del sistema stesso. Tale caratterizzazione richiede la definizione di una scala territoriale rispetto alla quale è possibile tradurre in termini concreti il modello teorico generale. Un aspetto molto delicato da questo punto di vista è proprio quello della scelta della dimensione territoriale, dato che questa deve rappresentare un riferimento spaziale non solo capace di circoscrivere delle dimensioni economiche, sociali e ambientali ma anche di cogliere le relazioni fra le strutture che sottendono i processi di produzione e consumo, il loro impatto ambientale e la generazione di benessere nella comunità.

Altro aspetto da considerare in chiave applicativa è quello della scelta degli indicatori per mezzo dei quali tentare di tradurre in termini quantitativi il modello proposto. Per quanto riguarda i flussi, si tratta di individuare, da un lato, un'adeguata misura del benessere sociale che sia applicabile per una comunità locale e, dall'altro, un metodo capace di valutare l'impatto ambientale ponendo in relazione, a livello territoriale, l'offerta di servizi ecosistemici con la domanda di risorse naturali da parte del sistema economico. Per gli stock, invece, è necessario operare una quantificazione delle strutture di produzione e di consumo che, tenendo conto della loro connotazione di agenti di trasformazione, deve guardare alla loro dimensione economica, sociale e valoriale.

Al termine di questo processo, i cui passaggi sono stati descritti nella prima parte della nota, è stato possibile tentare una applicazione esplorativa del modello con il quale affrontare una lettura territoriale delle relazioni fra dimensioni del sistema economico, livello di benessere e sostenibilità.

I risultati dell'analisi empirica, pur scontando degli innegabili limiti riguardo alla scelta degli indicatori, alle modalità utilizzate per il loro calcolo e alle difficoltà di generalizzazione di un singolo caso studio, sembrano fornire alcuni interessanti spunti di riflessione.

Il primo è rappresentato dalla notevole diversità che si osserva nelle caratteristiche economiche, sociali e ambientali di sistemi locali che insistono all'interno di un territorio accomunato da percorsi storici, substrati valoriali e stili di vita abbastanza simili. La lettura di tali differenze, se considerate alla luce delle interrelazioni che il modello pone alla base del funzionamento del sistema economico, offre utili indicazioni sui percorsi più adeguati per promuoverne uno sviluppo finalizzato al miglioramento del benessere delle comunità.

Si evidenzia, infatti, come non sia possibile, anche in un territorio di dimensioni ridotte come quello provinciale, proporre dei modelli univoci di sviluppo che prescindono dalle caratteristiche dei diversi sistemi locali, vale a dire dagli stock che ne definiscono la struttura e dai flussi che ne descrivono il funzionamento. A tale riguardo, viene da chiedersi, nella misura in cui l'obiettivo del sistema economico sia effettivamente la creazione del benessere sociale, in che modo gli attori 
istituzionali ed economici locali possano tenere conto di tali diversità e, soprattutto, quali azioni possano mettere in atto per indirizzare il territorio verso uno sviluppo che guardi prioritariamente a tale dimensione.

Il modello di analisi, grazie alla sua impostazione teorica, può contribuire a dare una parziale risposta a tali domande. Infatti, avendo individuato nella dimensione e nel funzionamento degli stock gli agenti causali della capacità del sistema di modificare le sue performance ambientali e sociali, è proprio sugli stock che devono concentrarsi le azioni di sviluppo locale.

$\mathrm{Se}$, in generale, questo discorso riguarda tutte le dimensioni strutturali economiche, sociali, valoriali - dei processi di produzione e di consumo, nel nostro caso ci si è focalizzati esclusivamente sugli stock che caratterizzano la dimensione produttiva del sistema locale. Con riferimento a questo aspetto, l'analisi ha evidenziato alcuni aspetti sui quali vale la pena di soffermarsi brevemente, in particolare per quanto riguarda le possibili implicazioni per gli indirizzi strategici delle imprese che operano nei diversi contesti territoriali.

Da questo punto di vista, le relazioni che emergono fra dimensione economica, sostenibilità ambientale e benessere della comunità locale dovrebbero spingere le imprese a pianificare e ad adottare strategie gestionali "compatibili" con le caratteristiche del territorio.

Così, nei sistemi locali in cui si osservano situazioni di ampia disponibilità di risorse naturali e scarsa dotazione produttiva, la via maggiormente percorribile per assicurare benessere alla comunità, il che in molti casi vuol dire anche evitare fenomeni di abbandono del territorio, appare quella dello sviluppo economico ottenuto anche attraverso un più intenso utilizzo dei servizi ecosistemici locali.

$\mathrm{Al}$ contrario, nei territori in cui si osserva un sistema produttivo sovradimensionato rispetto alle risorse naturali e inefficace, quando non controproducente, rispetto alla promozione della dimensione sociale, appare più ragionevole una strategia imprenditoriale tesa alla progressiva riduzione dello stock economico. Se nel breve periodo questa strategia può apparire contraria alle logiche di crescita economica dell'impresa, non può essere ignorato come rappresenti l'unica via per garantirne l'integrazione con la collettività e la permanenza di lungo periodo sul territorio.

$\mathrm{Ne}$ deriva, in conclusione, come sia importante per gli attori locali puntare su strategie gestionali che, guardando con attenzione alle caratteristiche, alle risorse e alle vocazioni del territorio, possano contribuire a uno sviluppo locale compatibile con la preservazione e la valorizzazione dell'ambiente e finalizzato a migliorare il benessere della comunità; è questa la forma di reale integrazione con il territorio che appare una condizione necessaria per rendere resilienti i sistemi economici locali, capaci cioè di affrontare e superare i sempre più frequenti shock che provengono dall'esterno. 


\section{Bibliografia}

ANGELI L., FRANCO S., SENNI S. (2001), "Ruralità e occupazione nelle province italiane, Un'analisi tipologica”, in Esposti R., Sotte F. (a cura di), Le dinamiche del rurale Letture del caso italiano, Franco Angeli, Milano.

BAGLIANI M., GALLI A., NICCOLUCCI V., MARCHETTINI N. (2008), "Ecological Footprint analysis applied to a sub-national area. The case of the province of Siena (Italy)", Journal of Environmental Management, n. 86, pp. 354-364.

BARBIERI G., PELLEGRINI G. (2001), "I sistemi locali del lavoro: uno strumento per la politica economica in Italia e in Europa", Atti del Convegno Uval-DPS, Ministero del Tesoro, Roma, pp. 242-256.

BLASI E., BONAIUTI M., FRANCO S., PANCINO B. (2008), "Modello a "stock e flussi" e governance dei sistemi locali", XLV Convegno della Società Italiana di Economia Agraria, Portici, 25-27 Settembre 2008.

BLASI E., PASSERI N. (2009), "La sostenibilità ambientale del sistema economico: il caso della Provincia di Viterbo", Tuscia Economica, n. 1, pp. 35-105.

BLASI E., PASSERI N., FRANCO S., PANCINO B., CICATIELlO C. (2010), "The contribution of sub-regional areas to local sustainability", in Bastianoni S. (a cura di) The state of the art in Ecological Footprint Theory and Application, Academic Conference, Footprint Forum 2010 - Short Communications, Siena.

BLASI E., NUCERA M., CICATIELLO C., FRANCO S. (2012), "Socio-demographic components of eudaimonic well-being: a survey in an italian province", Social Indicators Research, 14 June 2012, pp. 1-20.

BONAIUTI M. (2003), "Introduzione", in Georgescu Roegen N., Bioeconomia. Verso un'altra economia ecologicamente e socialmente sostenibile, Bollati Boringhieri, Torino.

BONAIUTI M. (2008), “Economia e territorio. Un approccio sistemico", Sviluppo locale, vol. 11, n. 27, pp. 32-56.

DIENER R., SUH E. (1997), "Measuring quality of life: economic, social and subjective indicators", Social Indicators research, n. 40, pp. 189-216.

ENDICOTT J., NEE J., HARRISON W., BLUMENTHAL R. (1993), "Quality of Life Enjoyment and Satisfaction Questionnaire: a new measure", Psychopharmacol Bullettin, vol. 29, n. 2, pp. 321-326.

EASTERLIN RA. (1974), "Does Economic Growth Improve the Human Lot?", in Paul A.D., Melvin W.R. (a cura di), Nations and Households in Economic Growth: Essays in Honor of Moses Abramovitz, Academic Press, New York.

FRANCO S., SENNI S. (2002), "Ruralità senza fratture: percorsi di sviluppo locale nella provincia di Viterbo", in Esposti R., Sotte F. (a cura di), La dimensione rurale delle sviluppo locale, Franco Angeli, Milano.

GEORGESCU-ROEGEN N. (1971), The entropy law and the economic process, Mass.: Harvard University Press, Cambridge.

GEORGESCU-ROEGEN N. (2003), Bioeconomia. Verso un'economia ecologicamente e socialmente sostenibile, Bollati Boringhieri, Torino.

ISTAT, IRPET (a cura di Sforzi F.) (1989), I mercati locali del lavoro in Italia, Franco Angeli, Milano.

ISTAT (2005), I sistemi locali del lavoro in Italia. Censimento 2001. Dati definitivi, ISTAT, 21 luglio 2005.

ISTAT (2008), Valore aggiunto e occupati interni per Sistema Locale del Lavoro. Anni 20012005, 10 luglio 2008. 
ISTAT (2012), Geo Demo. Demografia in cifre, consultabile online su http://demo.istat.it/.

MASLOW A.H. (1943), Motivation and Personality, Harper\&Row, (trad. in italiano) (1973), Motivazioni e Personalità, Armando Editore, Roma.

MATURANA H., VARELA F. (1980), Autopoiesi e cognizione, Marsilio, Venezia.

MINISTERO DELL'AMBIENTE, WWF (2004), L'Impronta Ecologica delle Regioni dell'Obiettivo 1 Del QCS del 2000/2006 - Un contributo per valutare lo sviluppo sostenibile, Ministero dell'Ambiente, Roma.

OECD (2002), OECD Redefining Territories, the functional regions, Paris.

PROVINCIA DI MILANO (2008), Impronta Ecologica della Provincia di Milano, consultabile online su www.provincia.milano.it.

REES W.E., WACKERNAGEL M. (1994), "Ecological footprints and appropriated carrying capacity: measuring the natural capital requirements of the human economy", in Jansson A., Hammer M., Folke C., Costanza R. (Eds.), Investing in natural capital: the ecological economics approach to sustainability, Island Press, Washington, pp. 362-390.

REES W., WACKERNAGEL M. (2008), L'impronta ecologica, come ridurre l'impatto dell'uomo sulla terra, Edizioni Ambiente, Roma, 2008.

RUINI C., OTTOLINI F., RAFANELLI C., RYFF C., FAVA G.A. (2003), "Italian validation of Psychological Well-being Scales (PWB)", Rivista di Psichiatria, vol. 38, n. 3, pp. 117-129.

RYAN R.M., DECI E.L. (2000), "Self-determination theory and the facilitation of intrinsic motivation, social development, and well-being", American Psychologist, n. 55, pp. 68-78.

RYFF C.D. (1989), "Happiness is everything, or is it? Explorations on the meaning of psychological well-being", Journal of Personality and Social Psychology, n. 57, pp. 1069-1081.

SCOTTI M., BONDAVALLI C., BODINI A. (2009), "Ecological Footprint as a tool for local sustanability: The municipality of Piacenza (Italy) as a case study", Environmental Impact Assessment Review, n. 29, pp. 39-50.

WACKERNAGEL M., REES W.E. (1996), Our ecological footprint. Reducing Human Impact on the Earth, New Society Publisher, Gabriola Island, Canada.

WWF ITALIA, WWF REGIONE TOSCANA, CRAS (2002), Ecological Footprint Regione Toscana - Calcolo dell'Impronta Ecologica della Regione Toscana, consultabile online su http://www.rete.toscana.it.

ZAMAGNI S. (1979), Georgescu-Roegen: $i$ fondamenti della teoria del consumatore, Etas Libri, Milano. 


\section{Appendice}

II questionario per la valutazione del benessere individuale

Quanto è d'accordo con ciascuna delle seguenti affermazioni?

1. Sono soddisfatto del mio stato di salute

\begin{tabular}{|c|c|}
\hline Per niente & Poco \\
\hline
\end{tabular}

\begin{tabular}{|c|c|c|c|c|}
\hline Per niente & Poco & Abbastanza & Molto & Del tutto \\
\hline
\end{tabular}

3. Ho spesso mal di testa o mal di pancia o soffro di insonnia

\begin{tabular}{|c|c|c|c|c|}
\hline Per niente & Poco & Abbastanza & Molto & Del tutto \\
\hline
\end{tabular}

4. Ho talmente tante cose da fare che non rimane tempo per me

\begin{tabular}{|c|c|c|c|c|}
\hline Per niente & Poco & Abbastanza & Molto & Del tutto \\
\hline
\end{tabular}

5. Provo spesso momenti di rabbia

\begin{tabular}{|c|c|c|c|c|}
\hline Per niente & Poco & Abbastanza & Molto & Del tutto \\
\hline
\end{tabular}

6. Di solito riesco a fare le cose che mi sono proposto

\begin{tabular}{|c|c|c|c|c|}
\hline Per niente & Poco & Abbastanza & Molto & Del tutto \\
\hline
\end{tabular}

7. Mi piace il lavoro che faccio

\begin{tabular}{|c|c|c|c|c|}
\hline Per niente & Poco & Abbastanza & Molto & Del tutto \\
\hline
\end{tabular}

8. Sono contento della mia vita sentimentale

\begin{tabular}{|c|c|c|c|c|}
\hline Per niente & Poco & Abbastanza & Molto & Del tutto \\
\hline \multicolumn{5}{|l|}{ ordo con i } \\
\hline Per niente & Poco & Abbastanza & Molto & Del tutto \\
\hline
\end{tabular}

10. Le mie giornate sono noiose

\begin{tabular}{|c|c|c|c|c|}
\hline Per niente & Poco & Abbastanza & Molto & Del tutto \\
\hline \multicolumn{5}{|c|}{ 11. Posso permettermi di comprare ciò che voglio } \\
\hline Per niente & Poco & Abbastanza & Molto & Del tutto \\
\hline
\end{tabular}

12. Sono soddisfatto del mio aspetto fisico

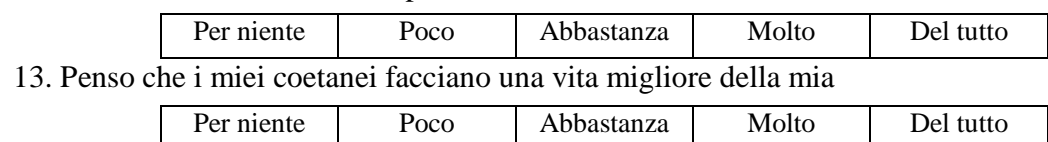

14. Penso che la mia vita sia in costante miglioramento

\begin{tabular}{|c|c|c|c|c|}
\hline Per niente & Poco & Abbastanza & Molto & Del tutto \\
\hline
\end{tabular}

15. Mi sento stimato dalle persone che mi circondano

\begin{tabular}{|l|l|l|l|l|}
\hline Per niente & Poco & Abbastanza & Molto & Del tutto \\
\hline
\end{tabular}

16. Nell'insieme sono soddisfatto della qualità della mia vita

\begin{tabular}{|c|c|c|c|c|}
\hline Per niente & Poco & Abbastanza & Molto & Del tutto \\
\hline
\end{tabular}

